\title{
Prevention of Perioperative Brachial Plexus Injury: A Modified Position
}

\author{
Ashutosh Kaushal ${ }^{1}$ \\ ${ }^{1}$ Department of Neuroanaesthesiology and Critical Care, \\ Neurosciences Centre, All India Institute of Medical Sciences \\ (AlIMS), New Delhi, India
}

J Neuroanaesthesiol Crit Care 2018;5:129-130

Brachial plexus injury is an important perioperative concern related to patient positioning. Digital subtraction angiography and radiological interventions of the thoracic spinal cord require the positioning of the upper limb such that it does not create any artifact during imaging of the spinal vasculature. The usual positioning of the arms during these procedures entails the risk of brachial plexus injury, though there are not much data available on it till date ${ }^{1}(\mathbf{- F i g}$. 1A). We suggest a new technique of patient positioning during endovascular procedure of upper thoracic spinal dural arteriovenous fistula, under fluoroscopy.

A 25-year-old man weighing $54 \mathrm{~kg}$ diagnosed to have upper thoracic dural arteriovenous fistula was scheduled for endovascular embolization. Preanesthetic evaluation was unremarkable. General anesthesia was induced with intravenous fentanyl $100 \mu \mathrm{g}$ and propofol $100 \mathrm{mg}$. Rocuronium $50 \mathrm{mg}$ was administered for muscle relaxation to facilitate tracheal intubation with an $8.5-\mathrm{mm}$ ID endotracheal tube.

\author{
Hemanshu Prabhakar ${ }^{1}$
}

Address for Correspondence Charu Mahajan, MD, DM, Department of Neuroanaesthesiology and Critical Care, Neurosciences Centre, AlIMS, New Delhi 110029, India (e-mail: charushrikul@gmail.com).

Anesthesia was maintained with sevoflurane in oxygen, nitrous oxide mixture and intermittent doses of rocuronium and fentanyl. The patient is usually positioned supine such that during fluoroscopy the arms remain away and do not create artifact in the field of interest. For this case, we adopted a new position in which the patient was placed supine with both the shoulders and elbows flexed at 90 degrees such that part of the forearm rests on the head and the wrist remains in neutral position. Upper extremities were strapped in this position with adequate soft protective padding between strap and elbow ( - Fig. 1B). Procedure was uneventful and lasted for 5 hours. Neuromuscular block was reversed, trachea extubated, and the patient was shifted to the ward at the end of procedure. Immediate postoperative course was uneventful. The patient did not have any sensory or motor loss nor did he complain of pain in shoulders during postoperative period. He was followed up till 5 days until discharge for home.

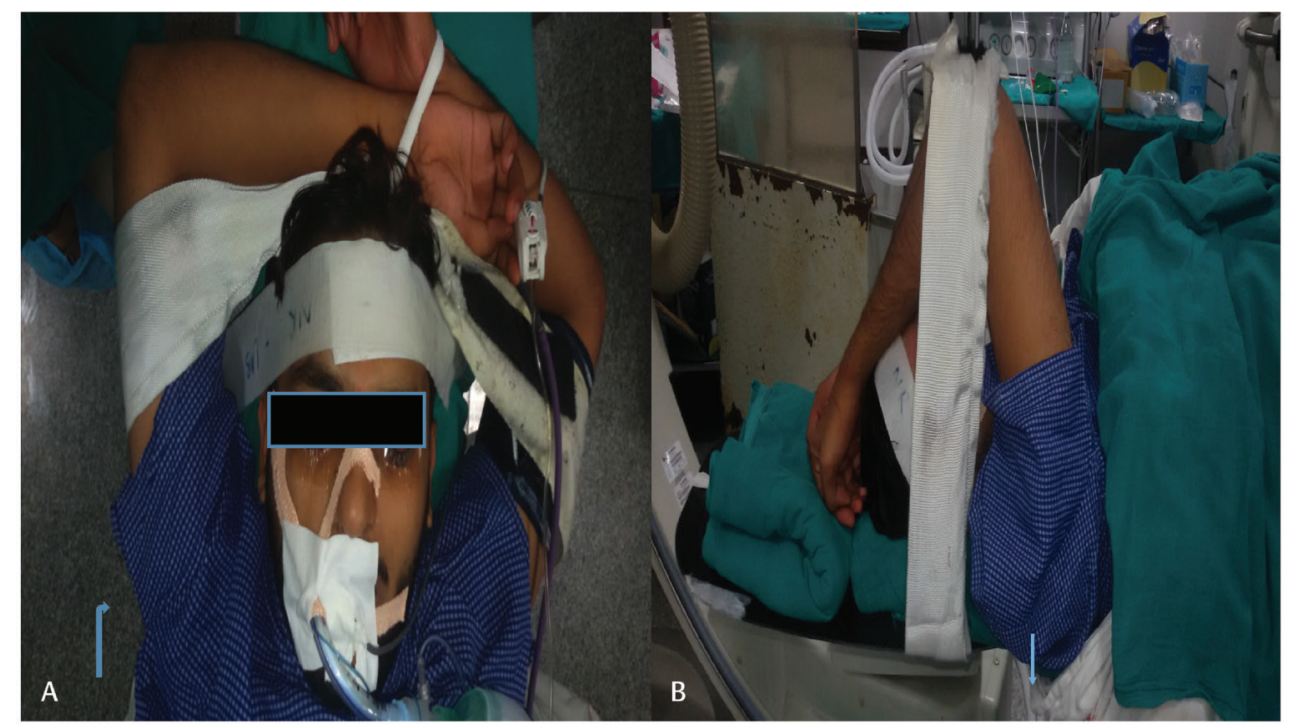

Fig. 1 (A) The usual position where arms need to be hyperabducted beyond 90 degrees for obtaining artifact-free fluoroscopic image of high thoracic lesions. (B) Proposed modified position wherein first both the shoulders are simultaneously flexed to 90 degrees and then both the elbows are also flexed so that part of the forearm rests on head and wrist remains in neutral position. The arms and forearms are strapped with a protective padding in a soft sling. This avoids hyperabduction at shoulders and minimizes the risk of brachial plexus injury.

received

April 21, 2018

accepted

May 07, 2018

published online

June 05, 2018
Copyright $\odot 2018$ Indian Society of Neuroanaesthesiology and Critical Care
License terms

10.1055/s-0038-1660529 ISSN 2348-0548.
(잉 $\Theta \circledast$ 
In an anesthetized patient, brachial plexus injury is the second most common upper extremity peripheral nerve injury due to inappropriate positioning of patient. ${ }^{2}$ American Society of Anesthesiologists Task Force on Prevention of Perioperative Peripheral Neuropathies suggest that abduction in a supine patient should be limited to 90 degrees for prevention of brachial plexus injury. ${ }^{3}$ Vertebral and prevertebral fasciae provide proximal attachment whereas axillary sheath provides distal attachment to brachial plexus. Overstretching due to exaggerated abduction of arm in anesthetized patient can lead to vasa vasorum injury, resulting in ischemia of brachial plexus. ${ }^{4}$ Usually the patient is positioned supine with arms in neutral position, tucked at side with protective padding or arms are folded across the chest during endovascular procedures under fluoroscopy. This position is appropriate for most of the endovascular procedures such as intracranial aneurysm coiling, intracranial arteriovenous malformation, carotid stenting, and lower thoracic and lumbar spinal malformation. However, in patients with upper and mid thoracic spinal malformations, upper extremities cannot be tucked at side as fluoroscopy in lateral view leads to superimposition of image over desired spinal malformation image. Also, in a supine patient with arms on an arm-rest, free movement of fluoroscope is hindered. Routine practice is to abduct the arm overhead with both shoulders flexed to 120 degrees and both elbows flexed to 90 degrees (-Fig. 1A). This leads to overabduction of the arms beyond 90 degrees, which is not acceptable as this position can lead to brachial plexus injury, especially during long-duration procedures as reported previously. ${ }^{5,6}$ To avoid this complication, we suggest a new patient position for these patients with arms and forearms flexed at 90 degrees with head and wrist in neutral position. However, there is a concern regarding invasive lines secured in upper limb. For an intravenous line to flow uninterrupted, the fluid bag has to be hanged at a greater height than usual for increasing the flow. Another alternative can be to use a pressure bag for enabling continuous flow of intravenous fluid. The arterial line, if indicated, may be inserted in dorsalis pedis or posterior tibial artery to avoid dampening.

Thus, our proposed modified position serves the advantage of avoidance of hyperabduction at shoulder joint and minimizes the risk of brachial plexus injury while ensuring uninterrupted fluoroscope movement.

\section{Conflict of Interest}

None.

\section{Funding}

None.

\section{References}

1 Hazarika A, Kumar S, Singh M. Abnormal hand position leading to possible brachial plexus injury during spinal interventional neuroradiology: are we aware of it? J Neuroanaesth Crit Care 2015;2:142-143

2 Sawyer RJ, Richmond MN, Hickey JD, Jarrratt JA. Peripheral nerve injuries associated with anaesthesia. Anaesthesia 2000;55(10):980-991

3 Practice advisory for the prevention of perioperative peripheral neuropathies. A report by the ASA task force on prevention of perioperative peripheral neuropathies. Anaesthesiology 2000;92:1168-1182

4 Desai DC, Uribe A, Lachman T. Brachial plexus injury due to compression: an alternate mechanism of injury: case report and review of the literature. Am Surg 1997;63(6):487-489

5 Pillai AK, Ferral H, Desai S, et al. Brachial plexus injury related to patient positioning. J Vasc Interv Radiol 2007; 18:833-834

6 Shankar S, Vansonnenberg E, Silverman SG, Tuncali K, Flanagan HL Jr, Whang EE. Brachial plexus injury from CT-guided RF ablation under general anesthesia. Cardiovasc Intervent Radiol 2005;28(5):646-648 